\title{
Sacituzumab govitecan in breast cancer
}

\author{
Xiaojun Wang, Jiongjie Chen, Hua Xiao, Yang Shi \\ Everest Medicines Ltd., Beijing, China \\ Contributions: (I) Conception and design: Y Shi; (II) Administrative support: Y Shi; (III) Provision of study materials or patients: J Chen; (IV) \\ Collection and assembly of data: J Chen; (V) Data analysis and interpretation: X Wang; (VI) Manuscript writing: All authors; (VII) Final approval of \\ manuscript: All authors. \\ Correspondence to: Yang Shi. Everest Medicines Ltd., Beijing 100020, China. Email: yang.shi@everestmedicines.com.
}

\begin{abstract}
Sacituzumab Govitecan (SG, IMMU-132) is an antibody-drug conjugate that links the irinotecan active metabolite, SN-38 to a humanized monoclonal antibody targeting Trop-2, a transmembrane calcium signal transducer. Trop-2 is highly expressed in epithelial cancer cells of multiple tumor types, including breast cancer. Therefore, the anti-Trop-2 monoclonal antibody allows for targeted delivery of SN-38 to tumor cells. SN-38 is membrane permeable and may elicit antitumor effects in adjacent tumor cells (bystander effect) before internalization of the antibody-drug conjugate through hydrolysis of the linker or by intracellular SN-38 release after internalization. SG has been approved to treat patients with triple-negative breast cancer (TNBC) and patients with urothelial cancer in US. In phase III confirmatory ASCENT trials, SG showed more significant efficacy benefit in TNBC patients who had received at least two prior systemic therapies than treatment of physician choice. Neutropenia and diarrhea are the most common adverse events and can be managed with supportive care. The incidence of treatment discontinuation due to adverse events was low $(<5 \%)$. Evidence has shown that SG should be considered as a new standard of care in patients with pretreated metastatic triple-negative breast cancer (mTNBC). Besides, SG has shown encouraging activity in patients with pretreated $\mathrm{HR}^{+} / \mathrm{HER} 2^{-} \mathrm{mBC}$ with a predictable, manageable safety profile. Here, in this review article, we summarize recent studies of SG for breast cancer treatment and provide a systematic review of the recently developed therapeutic approach.
\end{abstract}

Keywords: Antibody-drug conjugate (ADC); sacituzumab govitecan (SG); Trop-2; breast cancer

Received: 04 September 2021; Accepted: 30 October 2021; Published: 31 October 2021.

doi: $10.21037 /$ tbcr-21-28

View this article at: https://dx.doi.org/10.21037/tbcr-21-28

\section{Background}

Triple-negative breast cancer (TNBC) is a subtype of breast cancer that lacks expression of estrogen receptor (ER), progesterone receptor (PR) and human epidermal growth factor 2 receptor (HER2) on cancer cell surface. Approximately $15 \%$ of breast cancer (BC) patients were diagnosed with TNBC and had the worst prognosis among all subtypes of BC (1). Currently, chemotherapy remains to be the backbone for treatment of metastatic TNBC (mTNBC). However, standard chemotherapy is associated with low response rates (10\% to $15 \%)$ and short progression-free survival (PFS) (2 to 3 months) among patients with pretreated $\mathrm{mTNBC}$. In recent studies, several novel drugs including PD-1/PD-L1 inhibitors and PARP inhibitors showed promising results for the treatment of TNBC in front lines $(1,2)$.

Based on IMpassion 130, FDA approved the combinational treatment of Atezolizumab and nab-paclitaxel in patients with advanced PD-L1-positive (defined as PD-L1 stained tumor-infiltrating immune cells (IC) of any intensity covering $\geq 1 \%$ of the tumor area) TNBC as first-line treatment. The median PFS of Atezolizumab/nab-paclitaxel combinational treatment group was 7.5 months, while it was 5 months with nab-paclitaxel single treatment in PD-L1-positive subset (hazard ratio, 0.62; 95\% CI, 0.49-0.78; $\mathrm{P}<0.001$ ). Overall survival (OS) in the combinational treatment group was 25.0 months, while it was 15.5 months with nab-paclitaxel 
Table 1 Clinical results of targeted and immuno-therapeutic drugs

\begin{tabular}{|c|c|c|c|c|c|}
\hline Agent & MOA & Patients & Phase \& patient size & Key efficacy results & Main toxicity \\
\hline $\begin{array}{l}\text { Pembrolizumab + chemo } \\
\text { vs. chemo }\end{array}$ & Anti-PD-1 & $\begin{array}{l}\text { Untreated } \\
\text { mTNBC }\end{array}$ & III, 566 vs. 281 & PD-L1+: PFS, 9.7 vs. 5.6 months & $\begin{array}{l}\text { Gastrointestinal toxicity, } \\
\text { myelosuppression }\end{array}$ \\
\hline $\begin{array}{l}\text { Atezolizumab + Nab-pac } \\
\text { vs. Nab-pac }\end{array}$ & Anti-PD-L1 & $\begin{array}{l}\text { Untreated } \\
\text { mTNBC }\end{array}$ & III, 451 vs. 451 & $\begin{array}{l}\text { PD-L1+: PFS, } 7.5 \text { vs. } 5.0 \text { months; } \\
\text { OS, } 25 \text { vs. } 15.5 \text { months }\end{array}$ & $\begin{array}{l}\text { Alopecia, nausea, cough, } \\
\text { peripheral neuropathy }\end{array}$ \\
\hline
\end{tabular}

mTNBC, metastatic triple-negative breast cancer; PFS, progression-free survival; OS, overall survival.

single treatment in $\mathrm{PD}-\mathrm{L} 1-$ positive subset (hazard ratio, 0.62 ; $95 \%$ CI, 0.45-0.86) (3). Similarly, the phase III KEYNOTE-355 study evaluated the efficacy of pembrolizumab combined with chemotherapy in mTNBC as first-line treatment. Improvement in PFS was noted in patients with PD-L1-positive tumors (defined as a combined positive score $>10$ ) treated with immunotherapy plus chemotherapy versus chemotherapy alone (median PFS 9.7 vs. 5.6 months; hazard ratio, 0.65 ; 95\% CI, 0.49-0.86; one-sided $\mathrm{P}=0.0012$ ) (4).

In a phase III OlympiAD trial, the PARP inhibitor olaparib increased PFS compared with chemotherapy in patients with advanced HER2-negative BC with germline BRCA mutation (median PFS 7.0 vs. 4.2 months, hazard ratio, $0.58 ; 95 \%$ CI, 0.43-0.80; $\mathrm{P}<0.001$ ) (5). Similar results were also demonstrated with another PARP inhibitor, talazoparib in advanced BC patients with germline BRCA1 and BRCA2 mutations in the EMBRACA study (8.6 vs. 5.6 months; hazard ratio, $0.54 ; 95 \%$ CI, 0.41-0.71; $\mathrm{P}<0.001)(6)$. However, a majority of patients have disease progression after receiving PD-1/PD-L1 inhibitors and PARP inhibitors, and standard therapeutic options are limited to chemotherapy. The clinical results of targeted and immune-therapeutic drugs are shown in Table 1.

\section{Antibody-drug conjugates (ADC)}

Traditional alkylating agents, antimetabolites and antimitotic agents are main chemotherapy drugs (7). The emergence of antibody drugs such as HER $2 \mathrm{mAb}$ made combinational therapy between antibody and chemotherapy a more promising therapeutic approach for HER $2^{+}$breast cancer. With the continuous improvement of antibody technology, several ADC drugs have been approved for cancer therapy.
For the first-generation of ADCs, murine antibodies were highly immunogenic. Human anti-murine antibodies (HAMAs) have reduced efficacy and toxicity. Conventional cytotoxic drugs, including doxorubicin, methotrexate, mitomycin as payload, the active dose can be within the micromolar range and are mainly conjugated with the mouse monoclonal antibody by non-cleavable linkers (amide or succinimide) (8).

For the second generation of ADCs, chimeric, humanized or fully human antibodies have reduced immunogenicity. In addition, active doses of humanized antibody that couples with highly toxic drugs can be of pmol level. However, the second-generation ADCs have a narrow therapeutic window, mainly due to off-target toxicity, fast clearance, and competition with unconjugated antibodies. The secondgeneration ADC drugs have different drug antibody ratios (DAR) from 0-8. It usually shows low tolerance, high plasma clearance efficiency and low in vivo efficacy when DAR over 4. For example, the DAR of Brentuximab Vedotin is 4, the DAR of Ado-Trastuzumab emtansine DAR is 3.5, and the DAR of Inotuzumab Ozogamicin is 6 (9).

The key to the third-generation ADC drugs is the sitespecific binding, which ensures antibody conjugate drugs with a clear DAR. In addition, the third-generation ADC drugs have significantly improved therapeutic efficacy in terms of antibody optimization, linkers, and binding of small-molecule drugs. The representative drugs include sacituzumab govitecan (SG) and Fam-trastuzumab deruxtecan. The third-generation ADC, which coupled with humanized $\mathrm{mAb}$ at a higher ratio, uses fewer toxic drugs, allows higher doses of drugs to be delivered to tumor site and executes a bystander effect (10). The major differences from $1^{\text {st }}$ to $3^{\text {rd }}$ generation of ADC are shown in Table 2. 
Table 2 Differences from $1^{\text {st }}$ to $3^{\text {rd }}$ generation of antibody-drug conjugates

\begin{tabular}{llll}
\hline Variables & First generation & Second generation & Third generation \\
\hline Antibody & Murine antibodies & Human antibodies & Human antibodies \\
Payloads & Weak potency & High potency & Moderate or high potency \\
Conjugation strategy & Random coupling & Random coupling & Random or specific coupling \\
Linkers & Non-cleavable & Non-cleavable & Cleavable \\
Homogeneous (drug to antibody ratio) & Heterogeneous & Heterogeneous & Homogeneous \\
Bystander effect & None & None & Most Have \\
\hline
\end{tabular}

\section{Trop-2}

Trop-1, 2, 3, and 4 were first identified as transmembrane proteins expressed on the surface of normal and malignant trophoblast cells in 1981. Abnormally elevated expression of Trop-2 was found in a variety of cancer tissues regardless of the levels of its baseline expression in normal tissues (11). One possible mechanism of high expression of Trop- 2 in cancer is that transcription factors that regulate cancer cell progression, like WT1, can enhance the transcription and expression of Trop-2. Trop-2 modulates growth, invasion and proliferation of BC cells and is upregulated in breast cancer cells, making it an ideal target for novel therapies for breast cancer (12).

In a nude mouse model of MDA-MB-231 BC cells, Trop-2-targeted antigen-binding fragments (Fab) has been demonstrated to inhibit the occurrence and progression of breast cancer. Besides, another novel human Fab antibody for Trop-2 inhibits breast cancer growth in vitro and in vivo (13). IMMU-132 also known as SG is a Trop-2 targeting ADC conjugated with topoisomerase I inhibitor SN-38, which is an active metabolite of irinotecan and has shown anti-tumor effect in multiple cancer types including breast cancer. The SN-38 deliver into the tumor of IMMU-132 was 136 times more than that of irinotecan as demonstrated in a preclinical mouse model of allograft of breast cancer (14).

As a novel therapeutic target, Trop-2, an internalizing antigen/antibody, proprietary linker chemistry, and high drug payload has led to a high therapeutic index of IMMU132 with acceptable tolerability, with a less occurrence of diarrhea than irinotecan (15).

\section{SG (IMMU-132, Trodelvy $^{\circledR}$ )}

SG is a first-in-class Trop-2 ADC drug. It contains three parts: a humanized monoclonal antibody (hRS7), payload SN-38 and a linker. The monoclonal antibody binds to
Trop-2, the trophoblast cell-surface antigen. The payload SN-38 is a topoisomerase inhibitor metabolized from Irinotecan that prevents repair of DNA damage and leads to apoptosis and cell death. The monoclonal antibody and SN-38 are conjugated by a hydrolyzable linker with a drug to antibody ratio of 7.6. Preclinical studies have shown that SG targets and binds to Trop-2-expressing cancer cells, which is subsequently internalized by cells. Upon entry into the cell, SG is hydrolyzed to release $\mathrm{SN}-38$. SN-38 inhibits topoisomerase I activity by competitively binding to topoisomerase I, preventing its molecular function, which is involved in single strand break reconnection. Eventually, the accumulated DNA damage induces apoptosis of cancer cells. The key characteristics of SG are shown in Table 3.

In April 2020, FDA granted accelerated approval for the use of SG in mTNBC patients, who previously received at least two regimens for metastatic disease, based on clinical efficacy and safety data from a phase I/ II basket study (IMMU-132-01) (16). In September 2020, the primary results of phase III ASCENT clinical study confirmed these findings. Recently, based on the published results of ASCNET study, FDA granted a full approval to sacituzumab govitecan-hziy (Trodelvy) for the treatment of patients with unresectable locally advanced or metastatic triple-negative breast cancer (TNBC) who have previously received 2 or more systemic therapies, at least 1 of them for metastatic disease (17). In May 2021, SG also obtained the priority review for treatment of mTNBC by National Medical Products Administration (China NMPA). The milestone of SG's use in BC are shown in Table 4.

\section{Clinical data}

\section{IMMU-132-01 TNBC cobort}

IMMU-132-01 is a phase I/II basket study. In mTNBC (18) 
Table 3 The characteristics of sacituzumab govitecan

\begin{tabular}{|c|c|}
\hline Properties & Properties \\
\hline \multirow{2}{*}{ Antibody } & $\begin{array}{l}\text { Trop-2 is a newly discovered cancer protein, which is overexpressed in multiple epithelial cancers and regulates signaling } \\
\text { pathways involved in cancer cell proliferation and metastasis }\end{array}$ \\
\hline & The humanized mAb shows ADCC activity \\
\hline \multirow{2}{*}{ Payload } & Overcome cross-resistance to prior chemotherapy drugs \\
\hline & $\begin{array}{l}\text { Given its membrane-permeable nature, free intracellular SN-38 may disperse outside of cells and elicit antitumor effects to } \\
\text { adjacent tumor cells }\end{array}$ \\
\hline Linker & A high drug-to-antibody ratio, with an average of 7 to 8 molecules of SN-38 per antibody molecule \\
\hline
\end{tabular}

Table 4 Key milestone of sacituzumab govitecan in breast cancer

\begin{tabular}{ll}
\hline Time & Stone \\
\hline Jan. 2015 & FTD for mTNBC in USA \\
Feb. 2016 & Breakthrough therapy designation \\
May 2018 & Submission by BLA in USA (May) \\
Jul. 2018 & Acceptance and Priority Review granted by BLA in USA \\
Jan. 2019 & Received FDA Complete Response Letter \\
Dec. 2019 & Submission by BLA \\
Apr. 2020 & Accelerated approved \\
Apr. 2021 & Regular approved for mTNBC in US \\
May 2021 & Priority approval status of National Medical Products Administration (China) \\
\hline
\end{tabular}

FTD, fast track designation; mTNBC, metastatic triple-negative breast cancer.

cohort, a total of 108 patients received SG after at least two systemic treatments for mTNBC. On days 1 and 8 of every 21 days treatment cycle, patients were given SG $(10 \mathrm{mg} / \mathrm{kg})$ intravenously. Patients received a median of three previous systemic therapies, like taxanes and anthracyclines. The investigator-assessed objective response rate (ORR) was $33.3 \%$. The median duration of response was 7.7 months. The median progression-free survival was 5.5 months. The median overall survival were 13.0 months. Ninety-two of 108 patients (85\%) had Grade 3/4 adverse events (AEs). Most common adverse events related to SG was GI and hematological toxicities. A proportion of $6.5 \%$ of patients have Grade 3 febrile neutropenia and $8 \%$ patients have
Grade 3 diarrhea. Dose reduction occurred in 36 of the 108 patients $(33 \%)$. Three patients $(2.8 \%)$ discontinued treatment due to AEs.

\section{IMMU-132-01 HR+ cobort}

Fifty-four patients were enrolled in the HR+ cohort of IMMU-132-01 (19). All patients were received at least two kinds of systemic therapies in any setting, one of which must have been endocrine-based therapy. SG $(10 \mathrm{mg} / \mathrm{kg}$, I.V.) was applied to them on days 1 and 8 of a 21-day treatment cycle. Patients received a median of three previous endocrine therapy regimens in any setting and 
Table 5 Summarized clinical results in breast cancer with sacituzumab govitecan

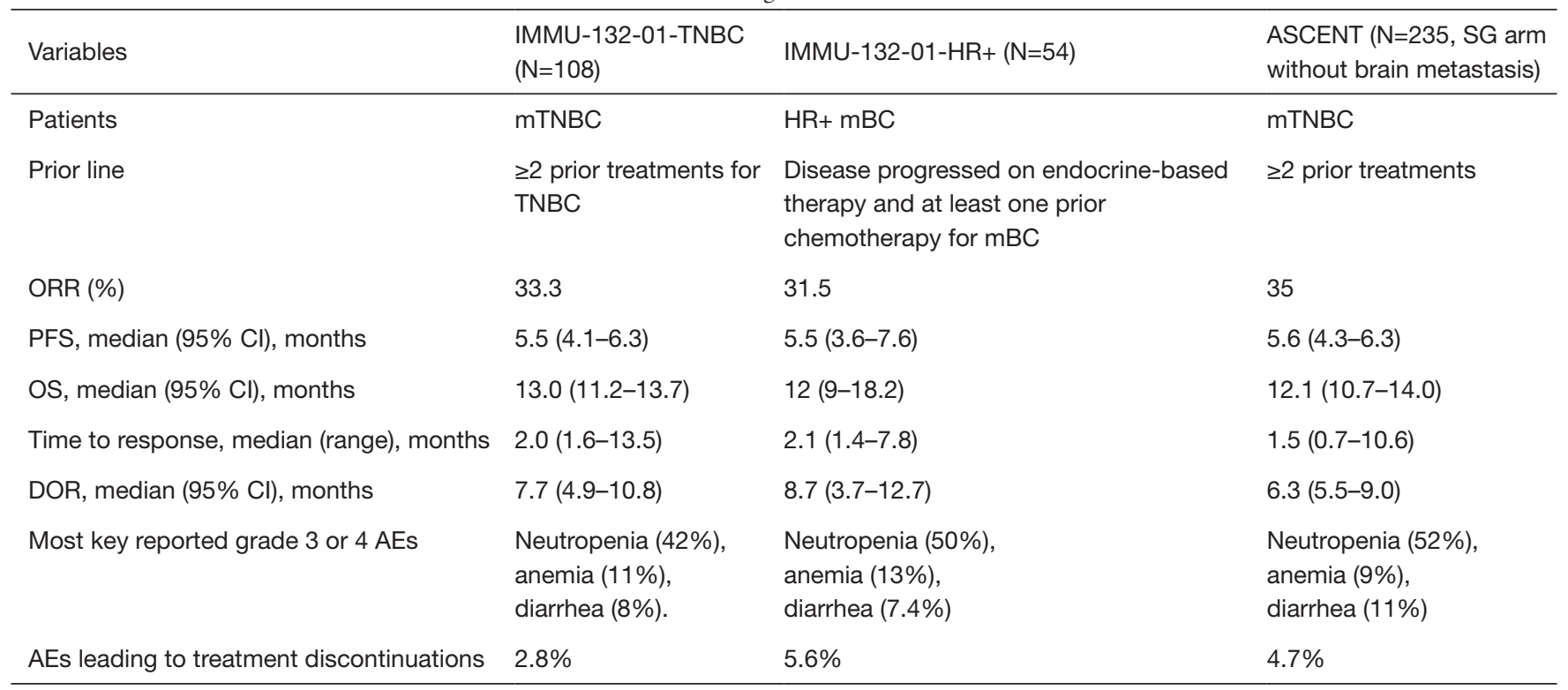

mTNBC, metastatic triple-negative breast cancer; ORR, objective response rate; PFS, progression-free survival; OS, overall survival; DOR, duration of response; $\mathrm{AE}$, adverse event.

median two prior chemotherapy regimens in metastatic setting. The mean follow-up time was 11.5 months. The ORR, median duration of response, median PFS, and median OS were $31.5 \%, 8.7$ months, 5.5 months, and 12 months, respectively. The most common grade $3 / 4$ treatment-related adverse events were neutropenia (50.0\%), anemia $(11.1 \%)$, and diarrhea $(7.4 \%)$. Two of the patients stopped the current therapy regimen due to treatment related AE. None of the patients died of SG related AE.

\section{ASCENT study}

The ASCENT study was an open label, randomized and controlled phase III trial (20). ASCENT investigated the efficacy and safety of SG versus the treatment of physician's choice (TPC). TPC is single-agent chemotherapy (eribulin, vinorelbine, capecitabine, capecitabine), a standard care in later liner of mTNBC. All patients were relapsed or refractory to two or more previous standard chemotherapy regimens (and without upper limit). The primary endpoint is PFS, assessed by independent central review (ICR). 468 patients without baseline brain metastases were enrolled. The mTNBC patients with disease-free interval less than 12 months after (neo)adjuvant chemotherapy would be counted as 1 prior treatment line. A heavily pre-treated patient population with a median number of 4 prior treatment lines (range, 2-17) were enrolled, including carboplatin and immune checkpoint inhibitors (ICIs) in $65.5 \%$ and $27.1 \%$ of patients without brain metastasis, respectively. The median progression-free survival was 5.6 months (95\% CI, 4.3-6.3; 166 events) with SG and 1.7 months (95\% CI, 1.5-2.6; 150 events) with chemotherapy (hazard ratio, $0.41 ; 95 \% \mathrm{CI}, 0.32-0.52 ; \mathrm{P}<0.001)$. The median overall survival was 12.1 months (95\% CI, 10.7-14.0) with SG and 6.7 months (95\% CI, 5.8-7.7) with chemotherapy (hazard ratio, 0.48; 95\% CI, 0.38-0.59; $\mathrm{P}<0.001$ ). The percentage of patients with an objective response was $35 \%$ with SG and 5\% with chemotherapy. The median duration of response was 6.3 and 3.6 months in SG group and TPC group, respectively. This trial was completed earlier than originally planned upon recommendation by an independent Data Safety Monitoring Committee because compelling evidence of efficacy regardless of BC related biomarkers, like TROP-2 expression and germline BRCA mutations had shown SG had a superior performance than TPC. The treatment-related adverse events were consistent with those previously reported. Neutropenia, alopecia, anemia, nausea, diarrhea and fatigue were the main treatment-related adverse events. $46 \%$ patients had alopecia. Neutropenia $(51 \%)$, diarrhea $(10 \%)$, anemia (8\%) and febrile neutropenia $(6 \%)$ were the 
Table 6 Summarized ongoing clinical trials in breast cancer with SG

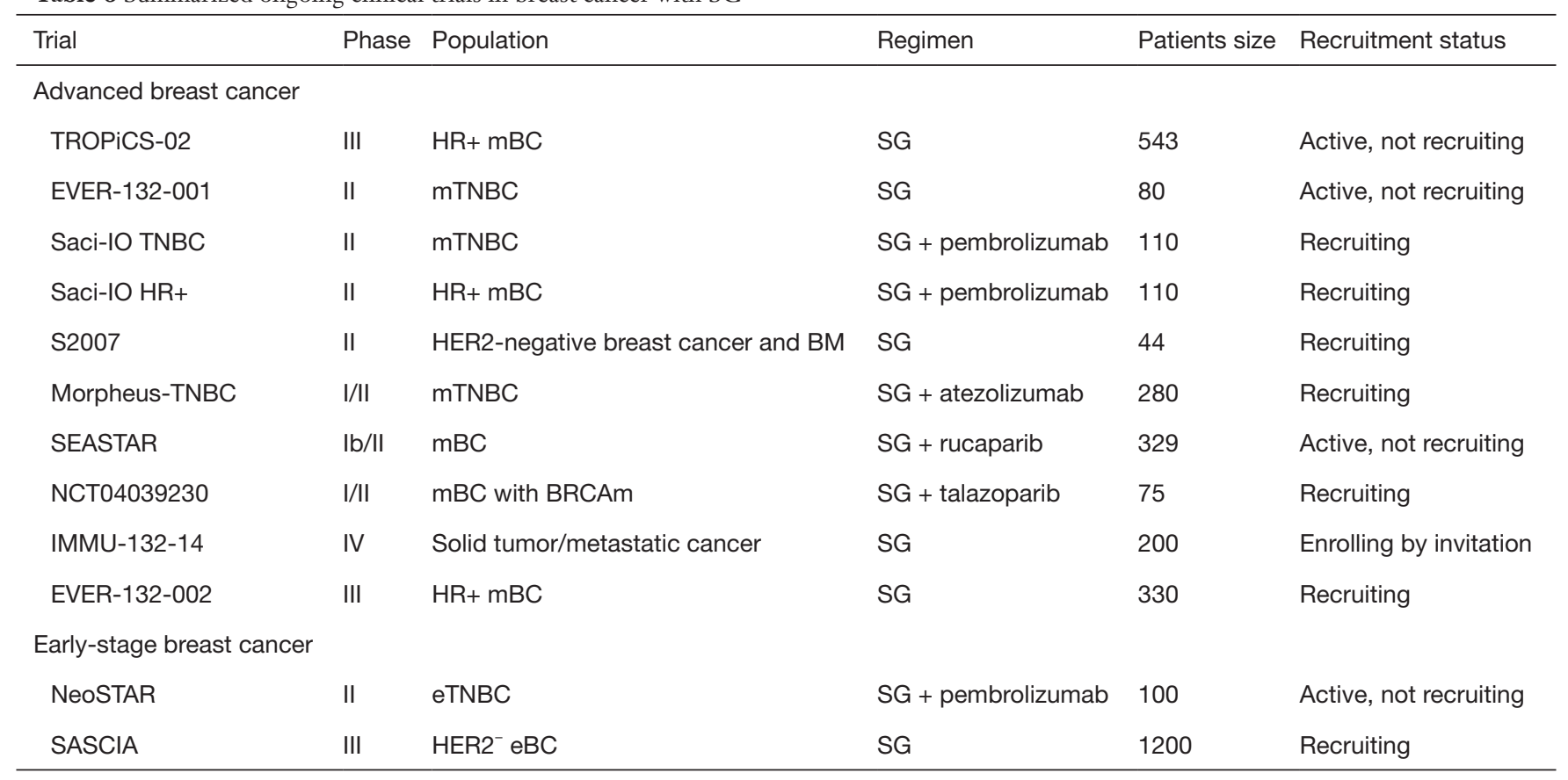

mTNBC, metastatic triple-negative breast cancer; SG, sacituzumab govitecan; BM, brain metastasis.

most common grade 3-4 treatment-related adverse events. The summarized clinical results in BC are shown in Table 5.

\section{Ongoing study}

A series of studies focusing on the treatment of breast cancer is ongoing. For patients with advanced stage cancer, two ongoing phase III clinical studies aim to evaluate the treatment efficacy of SG and TPC in patients with advanced $\mathrm{HR}+\mathrm{BC}$ [TROPiCS-02 (NCT03901339), EVER-132002 (NCT04639986)]. To explore the new combinational treatment, $\mathrm{SG}$ is under evaluation in combination with PD-1/PD-L1 inhibitors [Saci-IO TNBC (NCT04468061), Saci-IO HR+ (NCT04448886), Morpheus-TNBC (NCT03424005)] and PARPi [SEASTAR (NCT03992131), talazoparib (NCT04039230)]. For patients with earlystage breast cancer, SG is under phase III evaluation as post-neoadjuvant treatment in patients with early HER2$\mathrm{BC}$ presenting with residual disease after neoadjuvant treatment (SASCIA, NCT04595565). A neoadjuvant therapy [NeoSTAR (NCT04230109)] aiming to evaluate the efficacy of SG combined with PD-1 in early stage triple negative breast cancer is ongoing. The summarized ongoing clinical trials in BC with SG are shown in Table 6.

ADCs have demonstrated significantly improved efficacy and manageable toxicities as a new therapeutic approach for breast cancer. With the rapid development of ADC technology, it deserves further exploration. SG is a novel promising Trop-2 targeting ADC with potent anti-cancer activity showing in multiple epithelial cancer types, especially in breast cancer. Clinical evidence from phase III confirmatory RCT trial has supported that SG would be the standard of care in patients with pretreated mTNBC.

\section{Acknowledgments}

Funding: None.

\section{Footnote}

Conflicts of Interest: All authors have completed the ICMJE uniform disclosure form (available at https://dx.doi. org/10.21037/tbcr-21-28). All authors are from Everest Medicines Ltd. and they have no other conflicts of interest to declare.

Ethical Statement: The authors are accountable for all aspects of the work in ensuring that questions related to the accuracy or integrity of any part of the work are 
appropriately investigated and resolved.

Open Access Statement: This is an Open Access article distributed in accordance with the Creative Commons Attribution-NonCommercial-NoDerivs 4.0 International License (CC BY-NC-ND 4.0), which permits the noncommercial replication and distribution of the article with the strict proviso that no changes or edits are made and the original work is properly cited (including links to both the formal publication through the relevant DOI and the license). See: https://creativecommons.org/licenses/by-nc-nd/4.0/.

\section{References}

1. Zeichner SB, Terawaki H, Gogineni K. A Review of Systemic Treatment in Metastatic Triple-Negative Breast Cancer. Breast Cancer (Auckl) 2016;10:25-36.

2. Vidula N, Ellisen LW, Bardia A. Novel Agents for Metastatic Triple-Negative Breast Cancer: Finding the Positive in the Negative. J Natl Compr Canc Netw 2020. [Epub ahead of print]. doi: 10.6004/jnccn.2020.7600.

3. Schmid P, Adams S, Rugo HS, et al. Atezolizumab and Nab-Paclitaxel in Advanced Triple-Negative Breast Cancer. N Engl J Med 2018;379:2108-21.

4. Cortes J, Cescon DW, Rugo HS, et al. Pembrolizumab plus chemotherapy versus placebo plus chemotherapy for previously untreated locally recurrent inoperable or metastatic triple-negative breast cancer (KEYNOTE-355): a randomised, placebo-controlled, double-blind, phase 3 clinical trial. Lancet 2020;396:1817-28.

5. Robson M, Im SA, Senkus E, et al. Olaparib for Metastatic Breast Cancer in Patients with a Germline BRCA Mutation. N Engl J Med 2017;377:523-33.

6. Litton JK, Rugo HS, Ettl J, et al. Talazoparib in Patients with Advanced Breast Cancer and a Germline BRCA Mutation. N Engl J Med 2018;379:753-63.

7. Hafeez U, Parakh S, Gan HK, et al. Antibody-Drug Conjugates for Cancer Therapy. Molecules 2020;25:4764.

8. Beck A, Dumontet C, Joubert N. Antibody-drug conjugates in oncology. New strategies in development. Med Sci (Paris) 2019;35:1043-53.

9. Vankemmelbeke M, Durrant L. Third-generation

doi: $10.21037 /$ tbcr-21-28

Cite this article as: Wang X, Chen J, Xiao H, Shi Y. Sacituzumab govitecan in breast cancer. Transl Breast Cancer Res 2021;2:28. antibody drug conjugates for cancer therapy--a balancing act. Ther Deliv 2016;7:141-4.

10. Goldenberg DM, Sharkey RM. Sacituzumab govitecan, a novel, third-generation, antibody-drug conjugate (ADC) for cancer therapy. Expert Opin Biol Ther 2020;20:871-85.

11. Zaman S, Jadid H, Denson AC, et al. Targeting Trop2 in solid tumors: future prospects. Onco Targets Ther 2019;12:1781-90.

12. Shvartsur A, Bonavida B. Trop2 and its overexpression in cancers: regulation and clinical/therapeutic implications. Genes Cancer 2015;6:84-105.

13. Lin H, Zhang H, Wang J, et al. A novel human Fab antibody for Trop2 inhibits breast cancer growth in vitro and in vivo. Int J Cancer 2014;134:1239-49.

14. Sharkey RM, McBride WJ, Cardillo TM, et al. Enhanced Delivery of SN-38 to Human Tumor Xenografts with an Anti-Trop-2-SN-38 Antibody Conjugate (Sacituzumab Govitecan). Clin Cancer Res 2015;21:5131-8.

15. Cardillo TM, Govindan SV, Sharkey RM, et al. Sacituzumab Govitecan (IMMU-132), an Anti-Trop-2/ SN-38 Antibody-Drug Conjugate: Characterization and Efficacy in Pancreatic, Gastric, and Other Cancers. Bioconjug Chem 2015;26:919-31.

16. Available online: https://www.fda.gov/drugs/resourcesinformation-approved-drugs/fda-grants-acceleratedapproval-sacituzumab-govitecan-hziy-metastatic-triplenegative-breast-cancer. Accessed June 24, 2020.

17. Available online: https://www.fda.gov/drugs/resourcesinformation-approved-drugs/fda-grants-regular-approvalsacituzumab-govitecan-triple-negative-breast-cancer. Accessed May 10, 2021.

18. Bardia A, Mayer IA, Vahdat LT, et al. Sacituzumab Govitecan-hziy in Refractory Metastatic Triple-Negative Breast Cancer. N Engl J Med 2019;380:741-51.

19. Kalinsky K, Diamond JR, Vahdat LT, et al. Sacituzumab govitecan in previously treated hormone receptor-positive/ HER2-negative metastatic breast cancer: final results from a phase I/II, single-arm, basket trial. Ann Oncol 2020;31:1709-18.

20. Bardia A, Hurvitz SA, Tolaney SM, et al. Sacituzumab Govitecan in Metastatic Triple-Negative Breast Cancer. N Engl J Med 2021;384:1529-41. 